Article

\title{
Structural and Electronic Properties of Heterostructures Composed of Antimonene and Monolayer $\mathrm{MoS}_{2}$
}

\author{
Congcong Zhou ${ }^{1,+}{ }^{,}$Xiaodan $\mathrm{Li}^{1, *,+}$ and Taotao $\mathrm{Hu}^{2}$ \\ 1 College of Science, University of Shanghai for Science and Technology, Shanghai 200093, China; \\ 172282026@st.usst.edu.cn \\ 2 School of Physics, Northeast Normal University, Changchun 130024, China; hutt262@nenu.edu.cn \\ * Correspondence: xiaodan_li@usst.edu.cn; Tel.: +86-021-65691506 \\ $\dagger$ These authors contributed equally to this work.
}

Received: 23 October 2020; Accepted: 23 November 2020; Published: 27 November 2020

\begin{abstract}
Antimonene is found to be a promising material for two-dimensional optoelectronic equipment due to its broad band gap and high carrier mobility. The van der Waals heterostructure, as a unique structural unit for the study of photoelectric properties, has attracted great attention. By using $\mathrm{ab}$ initio density functional theory with van der Waals corrections, we theoretically investigated the structural and electronic properties of the heterostructures composed of antimonene and monolayer $\mathrm{MoS}_{2}$. Our results revealed that the $\mathrm{Sb} / \mathrm{MoS}_{2}$ hetero-bilayer is an indirect semiconductor with type-II band alignment, which implies the spatial separation of photogenerated electron-hole pairs. Due to the weak van der Waals interlayer interactions between the adjacent sheets of the hetero-bilayer systems, the band structures of isolated antimonene and monolayer $\mathrm{MoS}_{2}$ are preserved. In addition, a tunable band gap in $\mathrm{Sb} / \mathrm{MoS}_{2}$ hetero-bilayer can be realized by applying in-plane biaxial compressing/stretching. When antimonene and monolayer $\mathrm{MoS}_{2}$ are stacked into superlattices, the indirect semiconductors turn into direct semiconductors with the decreased band gaps. Our results show that the antimonene-based hybrid structures are good candidate structures for photovoltaic devices.
\end{abstract}

Keywords: $\mathrm{Sb} / \mathrm{MoS}_{2}$ hetero-bilayer; electronic properties; type-II band alignment

\section{Introduction}

Due to its unique properties and potential applications, graphene, a two-dimensional (2D) material honeycomb lattice composed of $\mathrm{C}$ atoms, has caused a tremendous upsurge of scientific interest [1-4]. Graphene and its polymers have excellent photoelectric and chemical properties, which are widely used in transistor and photoelectric devices [5-12]. However, graphene is a zero-band gap semiconductor with poor current switching ability, which limits its application in photoelectric devices [13]. Since the band gap is an essential property for logic circuit of field-effect transistors [14], we should find the substitutional materials for graphene. For example, boronnitride (BN) is a wide-band gap insulating material with good electrical insulation, high thermal conductivity and excellent lubricant properties [15-17]. Molybdenum disulfide $\left(\mathrm{MoS}_{2}\right)$, a typical layered-transition metal halide material, has been used widely in gas-sensing fields due to its high chemical response and excellent electronic properties [18-20]. Through mechanical exfoliation, the single layers of antimony can be readily produced $[21,22]$. The bilayer antimonene is shown to have enhanced stability and superconductivity, which is essential for the Li-ion battery [23-25]. However, with the rapid development of optoelectronic and new energy fields, a higher demand has been presented in 2D materials. Since single-structure 
materials are unable to meet the increasing demand of the material field, it is particularly important to study hybrid systems composed of different 2D materials.

Recent studies have shown that the two-dimensional components of hybrid systems could complement each other, which would provide more opportunities for achieving the desired optoelectronic properties. The electronic properties of hybrid systems change with the system's ingredients, resulting from the combination of van der Waals interactions and interlayer characteristics. For example, due to the introduction of $\mathrm{MoS}_{2}$, the charge mobility and the energy band of $\mathrm{MoS}_{2} / \mathrm{Si}$ and $\mathrm{MoS}_{2} / \mathrm{Ge}$ hybrid systems are increased, enhancing the application of electronic and optical properties in multifunctional devices [26-29]. A strong interlayer coupling between $\mathrm{MoS}_{2}$ and graphene was found in $\mathrm{MoS}_{2}$ /graphene heterostructures [30,31]. The coupling effect of $\mathrm{BN}$ with other components (graphene, graphite or metal) is strong, which means BN-based heterostructures can be used as a stable, high-purity and high-performance nano-scale dielectric material [32-35]. Moreover, a type-II band alignment was found in $\mathrm{GaSe} / \mathrm{MoSe}_{2}$ [36], black phosphorene/MoS 2 [37], Sb/graphene [38] and $\mathrm{Sb} /$ selenide [39] heterostructures. The type-II band arrangement can transfer holes and electrons to different materials, thus reducing the exciton recombination and increasing the exciton lifetime.

In contrast to flat graphene, antimonene is a pleated semiconductor with a finite band gap value and higher carrier mobility. In addition, the band gap of antimonene changes with the thickness, and it has higher chemical stability in the air. Considering the high sensitivity of antimonene, in this work, using density functional calculations with van der Waals correction, we systematically investigated the structural and electronic properties of hybrid systems composed of $\mathrm{MoS}_{2}$ and antimonene. We focus on the charge distribution and bonding nature in the hybrid systems, which would be helpful for a deeper understanding of common rules in electronic properties and type-II band gap control of hybrid systems. Our project helps in moving from theoretical results to experimental simulation, which can provide a theoretical foundation for the application of monolayer $\mathrm{MoS}_{2}$-based optoelectronic devices.

\section{Methods}

First-principles calculations were performed using the projector augmented wave (PAW) method within the density functional theory (DFT) [40] as implemented in the Vienna ab initio simulation package (VASP) [41,42]. The generalized gradient approximation (GGA) was used to deal with the electron interaction correlation potential [43]. In addition, in order to better explain the weak van der Waals interactions, which is crucial for layered heterostructures, all calculations in this work were performed using the PBE-D2 method [44,45]. In order to avoid the corresponding interlayer coupling, a vacuum layer of $15 \AA$ in the $z$ direction was set between two adjacent hetero-bilayers. The Brillouin zone integrations were approximated using the special K-point sampling of the Monkhorst-Pack scheme $[46,47]$ with a $\Gamma$-centered $15 \times 15 \times 1$ grid. Wave functions were expanded in plane waves up to a kinetic energy cutoff of $500 \mathrm{eV}$. The cell parameters and the atomic coordinates of all layered heterostructures are fully relaxed until the change of the energy and the force reaches $10^{-5} \mathrm{eV}$ per supercell and $10^{-2} \mathrm{eV} / \AA$, respectively. Although spin-polarized calculations were performed, the results indicated that all the layered heterostructures do not exhibit magnetism at their equilibrium states.

\section{Results and Discussion}

According to our results performed using the PBE-D2 method, the lattice constants of a free-standing monolayer $\mathrm{MoS}_{2}$ and antimonene are $3.19 \AA$ and $4.06 \AA$, respectively, which are in good agreement with the reported value [48-52]. Although the lattice constant of antimonene differs significantly from that of single-layer $\mathrm{MoS}_{2}$, they do share the same primitive cell of a hexagonal structure. In order to reduce the lattice mismatch between the antimonene and the $\mathrm{MoS}_{2}$ monolayer, the hybrid system was modeled with a supercell consisting of $3 \times 3$ unit cells of antimonene and $4 \times 4$ unit cells of $\mathrm{MoS}_{2}$ monolayer in the $x-y$ plane. The lattice constants of $3 \times 3$ antimonene and $4 \times 4 \mathrm{MoS}_{2}$ are $12.18 \AA$ and $12.76 \AA$, respectively, which leads to a $4.6 \%$ lattice mismatch initially in the $\mathrm{Sb} / \mathrm{MoS}_{2}$ hetero-bilayer. Compared with the heterostructures investigated previously, this lattice 
mismatch value is relatively small [53-55]. The optimized structure of the antimonene and single layer $\mathrm{MoS}_{2}$ are shown in Figure 1. Both A and B represent the geometric structure of antimonene, and $\mathrm{B}$ was obtained by rotating the structure $\mathrm{A}$ by $60^{\circ}$. C is the geometric structure of $\mathrm{MoS}_{2}$ monolayer. Through the weak VDW force, the antimonene can form a multilayer structure. The bilayer antimonene structures labeled as AA and AB are shown in Figure 1b. AA is a hexagonal stacking model, in which all $\mathrm{Sb}$ atoms in the upper layer $(\mathrm{A})$ are located above the $\mathrm{Sb}$ atom in the lower layer $(\mathrm{A})$. $\mathrm{AB}$ is the Bernal stacking model, in which the B layer is constructed from a rotation of the A layer by $60^{\circ}$. For the hetero-bilayer structure composed of antimonene and the $\mathrm{MoS}_{2}$ monolayer (marked as $\mathrm{Sb} / \mathrm{MoS}_{2}$ ), two types of stacking models were considered: (1) hollow-site stacking (denoted by AC), the center of antimony hexagons on top of one of the Mo or S atoms of $\mathrm{MoS}_{2}$ monolayer; and (2) top-site stacking (denoted by BC), a Sb atom of antimonene on top of one of the Mo or S atoms of the $\mathrm{MoS}_{2}$ monolayer. The side views of the bilayer antimonene and the $\mathrm{Sb} / \mathrm{MoS}_{2}$ hetero-bilayer are shown in Figure 1c.

(a)

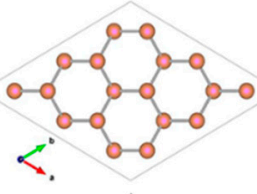

A

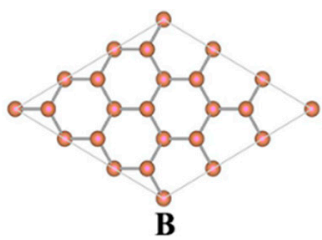

B

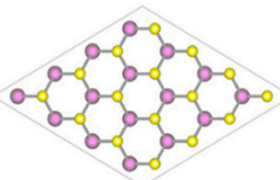

C

(b)

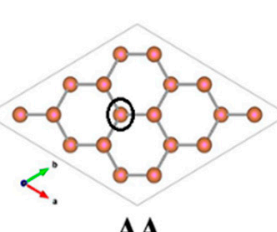

AA

c)

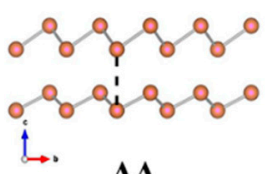

AA
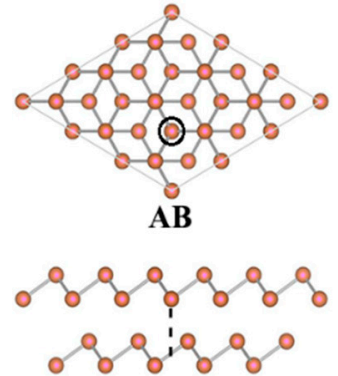

AB

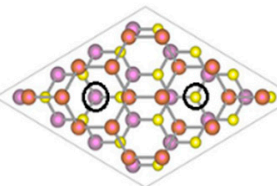

AC

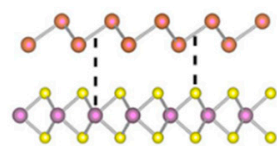

AC
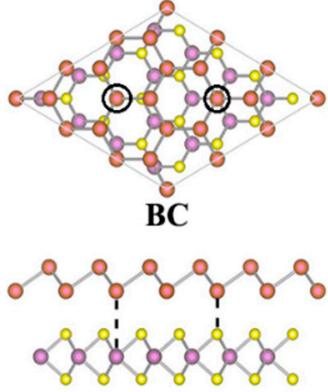

BC

Figure 1. (Color online) (a) Top view of the antimonene and $\mathrm{MoS}_{2}$ monolayer; (b) top view and (c) side view of the bilayer antimonene and the $\mathrm{Sb} / \mathrm{MoS}_{2}$ hetero-bilayer. $\mathrm{Sb}, \mathrm{Mo}$ and $\mathrm{S}$ atoms are represented by red, purple and yellow balls, respectively.

The optimized structural parameters of the bilayer antimonene are shown in Table 1 . The lattice constant " $a$ " of the AA and AB stacking is $12.73 \AA$ and $12.28 \AA$, respectively. Compared to the isolated antimonene, the AA stacking structure is stretched by $4.5 \%$ and the AB stacking structure is stretched by $0.8 \%$. The results show that there is a significant difference in the lattice constant between two stacking bilayer antimonene, which is caused by the increased coupling between layers. A shorter interlayer distance was found in the $\mathrm{AB}$ stacking bilayer antimonene, indicating that the interlayer interaction of the $\mathrm{AB}$ stacking structure is stronger. This can also explain why the amplitude of buckling $(\Delta)$ of the AA stack is slightly larger than the AB stack. To discuss the relative stabilities of the bilayer systems, all the binding energy $\left(E_{b}\right)$ in this paper is defined as:

$$
E_{b}=\left(E_{A}+E_{B}\right)-E_{A B}
$$

where $E_{A}$ and $E_{B}$ represent the total energies of the isolated monolayers and $E_{A B}$ is the total energy of the heterostructure. The binding energies are given per unit cell. As shown in Table 1, the binding energy of bilayer antimonene and $\mathrm{Sb} / \mathrm{MoS}_{2}$ hetero-bilayers are positive. This indicates that the layer stack is an exothermic process; in other words, the two-layer system discussed in this paper can exist stably. In addition, the small binding energy also indicates the weak interaction between the antimonene and $\mathrm{MoS}_{2}$ layers. The binding energy of bilayer antimonene optimized via the PBE-D2 method is 
$2.275 \mathrm{eV}$ and $3.513 \mathrm{eV}$ for the $\mathrm{AA}$ and $\mathrm{AB}$ stacking model, respectively (see Table 1), indicating that the interlayer interaction of the $\mathrm{AB}$ stacking is stronger than that of the AA stacking.

Table 1. Geometries and binding energies of antimonene and $\mathrm{MoS}_{2}$ monolayer, antimonene bilayer, $\mathrm{Sb} / \mathrm{MoS}_{2}$ hetero-bilayers and $\mathrm{Sb} / \mathrm{MoS}_{2}$ superlattice, including the bond length, interlayer distance of stacked layer structure $(\mathrm{h})$ and the amplitude of bucking of the antimonene layers $\left(\Delta_{S b}\right)$.

\begin{tabular}{cccccccc}
\hline System & & $\mathbf{a}(\AA)$ & $\mathbf{E}_{\mathbf{b}}(\mathbf{e V})$ & $\mathbf{d}_{\mathbf{S b}-\mathbf{S b}}(\AA)$ & $\mathbf{d}_{\mathbf{M o}-\mathbf{S}}(\AA)$ & $\mathbf{h}(\AA)$ & $\boldsymbol{\Delta}_{\mathbf{S b}}(\AA)$ \\
\hline Antimonene & & 12.18 & $/$ & 2.88 & $/$ & $/$ & 0.110 \\
\hline Monolayer $\mathrm{MoS}_{2}$ & & 12.76 & $/$ & $/$ & $/$ & $/$ & $/$ \\
\hline bilayer antimonene & $\mathrm{AA}$ & 12.73 & 2.275 & 2.95 & $/$ & 3.03 & 0.110 \\
\cline { 2 - 8 } & $\mathrm{AB}$ & 12.28 & 3.513 & 2.88 & $/$ & 2.90 & 0.107 \\
\hline $\begin{array}{c}\text { Sb/MoS } \\
\text { hetero-bilayer }\end{array}$ & $\mathrm{AC}$ & 12.67 & 1.377 & 2.92 & 2.42 & 3.41 & 0.074 \\
\cline { 2 - 7 } & $\mathrm{BC}$ & 12.66 & 1.378 & 2.92 & 2.41 & 3.41 & 0.075 \\
\hline $\begin{array}{c}\text { Sb/MoS } \\
\text { superlattice }\end{array}$ & $\mathrm{AC}$ & 12.67 & 3.612 & 2.92 & 2.42 & 3.29 & 0.137 \\
\cline { 2 - 7 } & $\mathrm{BC}$ & 12.66 & 3.661 & 2.91 & 2.40 & 3.34 & 0.147 \\
\hline
\end{tabular}

In order to better understand the properties of antimonene-based hybrid structures, we also studied the hetero-bilayer (labeled as $\mathrm{Sb} / \mathrm{MoS}_{2}$ ) and superlattice (labeled as $\mathrm{Sb} / \mathrm{MoS}_{2}$ ) composed of antimonene and monolayer $\mathrm{MoS}_{2}$. For hybrid systems, to quantitatively characterize the interlayer interaction, the binding energy between the antimonene and $\mathrm{MoS}_{2}$ sheet was calculated using the adsorption energy formula. $E_{A B}$ is the total energy of the $\mathrm{Sb} / \mathrm{MoS}_{2}$ hetero-bilayer and $E_{A} / E_{B}$ is the total energy of the isolated $\mathrm{Sb}$ sheet or monolayer $\mathrm{MoS}_{2}$. Similar to bilayer antimonene, for hybrid systems, we also considered two representative arrangements of antimonene on monolayer $\mathrm{MoS}_{2}$ (AC and BC). The optimized lattice constant " $a$ " of the $\mathrm{Sb} / \mathrm{MoS}_{2}$ hetero-bilayer is $12.67 \AA$ and $12.66 \AA$, respectively. Judging from the optimized structural parameters and electronic structures of hetero-bilayer $\mathrm{Sb} / \mathrm{MoS}_{2}$, there is no big difference between the $\mathrm{AC}$ and $\mathrm{BC}$ stacking structures (shown in Figure 1). These results indicate that the energetics of antimonene on $\mathrm{MoS}_{2}$ are not sensitive to the stacking models, which is similar to that of previous $\mathrm{MoS}_{2}$-based hybrid systems [29-31]. Compared to the corresponding isolated sheet, the $\mathrm{Sb}$ layer in the $\mathrm{Sb} / \mathrm{MoS} 2$ hetero-bilayer is stretched by $3.94 \%$ (from 4.06 to $4.22 \AA$ ), while the $\mathrm{MoS}_{2}$ layer in the $\mathrm{Sb} / \mathrm{MoS}_{2}$ heterogeneous double is compressed by $0.63 \%$ (from 3.19 to $3.17 \AA$ ).

Figure 2 illustrates the electronic band structures of the bilayer systems. For monolayer antimonene, the maximum valence band (VBM) is located at the high symmetry $\Gamma$ point, and the conduction band minimum (CBM) is located between the high symmetry point $\Gamma$ and $\mathrm{M}$. This indicates that the $\mathrm{Sb}$ monolayer is an indirect band gap semiconductor with a band gap of $1.25 \mathrm{eV}$, which is in good agreement with the reported value [21-25]. Moreover, for the $\mathrm{MoS}_{2}$ monolayer (shown in Figure 2b), both VBM and CBM are located between the high symmetry points $\Gamma$ and $\mathrm{M}$, suggesting that the $\mathrm{MoS}_{2}$ monolayer is a direct band gap semiconductor. The electronic band structures of bilayer antimonene with two different stacking models are shown in Figure 2c,d. We can see that the VBM of the AA stacked is located between the high symmetry points $\Gamma$ and $\mathrm{K}$, and the CBM is located at the high symmetry point $\Gamma$ from Figure 2c. However, the CBM and VBM of the AB stacked are both located between the high symmetry points $\Gamma$ and $\mathrm{M}$. Therefore, the bilayer antimonene is an indirect band gap semiconductor with a band gap of $0.36 \mathrm{eV}$ (AA stacking) and $0.25 \mathrm{eV}$ (AB stacking). The band gap value of the bilayer antimonene is reduced compared to the $\mathrm{Sb}$ monolayer, which is consistent with other van der Waals layered structures [22-25]. Compared to the $\mathrm{Sb}$ monolayer, the band gap value of the bilayer antimonene is significantly sunken regardless of the model stack. This facilitates the charge transfer between layers and realizes the application of $\mathrm{Sb}$ in photovoltaic cells. 
(a)

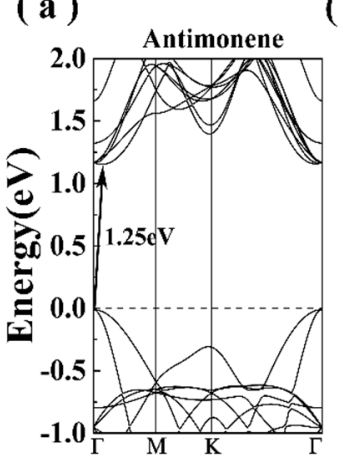

(e)

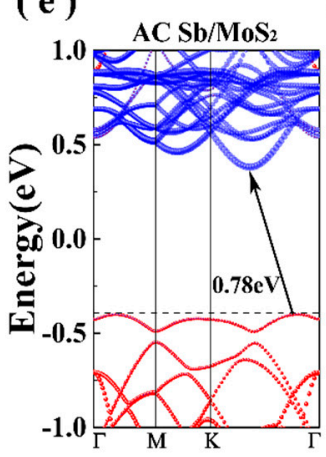

(b)

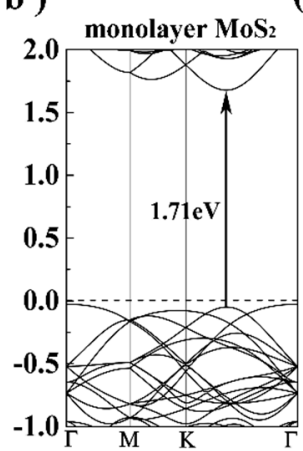

(c)

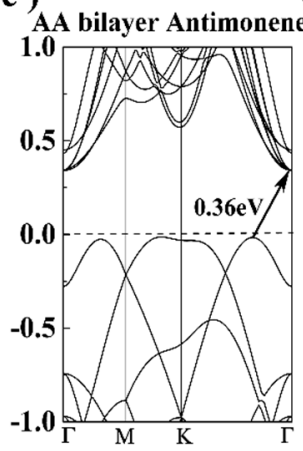

(d)

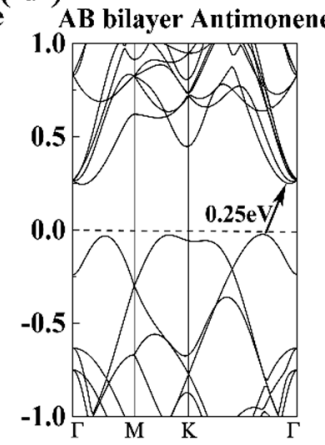

(f)

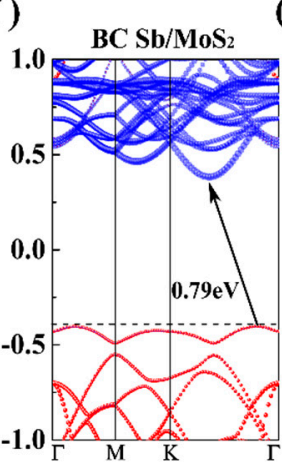

(g)

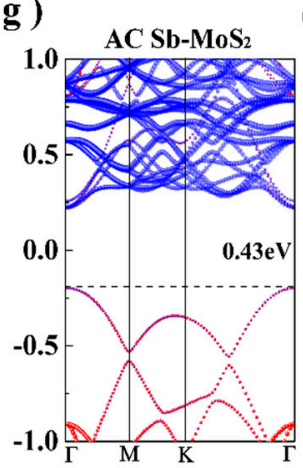

(h)

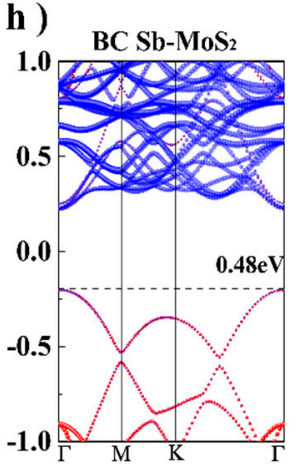

(i)

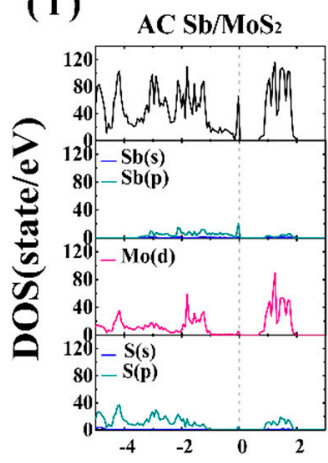

(j)

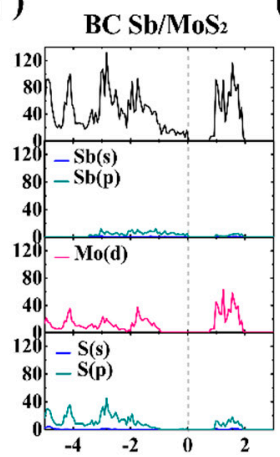

(k)

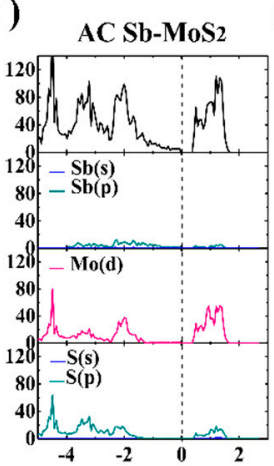

(1)

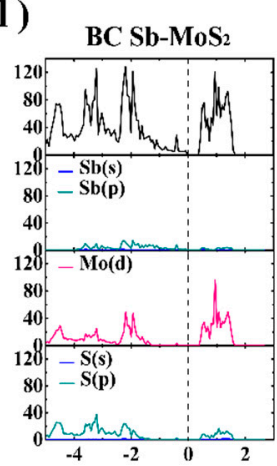

Figure 2. Band structures of (a) antimonene, (b) $\mathrm{MoS}_{2}$ monolayer, (c) AA bilayer antimonene, (d) AB bilayer antimonene, (e) AC Sb/MoS 2 hetero-bilayer, (f) BC Sb/MoS 2 hetero-bilayer, (g) AC Sb/MoS 2 superlattice and (h) BC Sb/MoS 2 superlattice. The density of states of (i) AC Sb/MoS 2 hetero-bilayer, (j) $\mathrm{BC} \mathrm{Sb} / \mathrm{MoS}_{2}$ hetero-bilayer, (k) $\mathrm{AC} \mathrm{Sb} / \mathrm{MoS}_{2}$ superlattice and (1) $\mathrm{BC} \mathrm{Sb} / \mathrm{MoS}_{2}$ superlattice. The black dotted line indicates the Fermi level.

The projected band structures of hetero-bilayer $\mathrm{Sb} / \mathrm{MoS}_{2}$ are shown in Figure 2e,f, the red and blue circles representing the contributions of $\mathrm{Sb}$ and $\mathrm{MoS}_{2}$ to the eigenstate, respectively. We can clearly see that the band structure of the $\mathrm{Sb} / \mathrm{MoS}_{2}$ heterostructure is a simple superposition of the components. There is a simple coupling effect between $\mathrm{Sb}$ and $\mathrm{MoS}_{2}$ layers so that the electronic structure of $\mathrm{Sb}$ and $\mathrm{MoS}_{2}$ monolayer can be preserved. Both for $\mathrm{AC}$ and for $\mathrm{BC}$ stacking, the CBM and VBM are located between the high symmetry points $\Gamma$ and $\mathrm{M}$, indicating that the $\mathrm{Sb} / \mathrm{MoS}_{2}$ hetero-bilayer is an indirect band gap semiconductor. In addition, the $\mathrm{VBM}$ of the $\mathrm{Sb} / \mathrm{MoS}_{2}$ heterostructure is mainly distributed from $\mathrm{Sb}$, while the CBM is localized in $\mathrm{MoS}_{2}$. Therefore, the $\mathrm{Sb} / \mathrm{MoS}_{2}$ is a type-II hetero-bilayer structure. In the $\mathrm{Sb} / \mathrm{MoS}_{2}$ type-II energy band alignment, $\mathrm{MoS}_{2}$ acts as an electron acceptor and $\mathrm{Sb}$ acts as an electron donor. According to the density of states (DOS) of the $\mathrm{Sb} / \mathrm{MoS}_{2}$ hetero-bilayer shown in Figure $2 i, j$, the energy band near the Fermi level originates from the electron hybridization of the $p$ orbital of $\mathrm{Sb}$ and the $d$ orbital of Mo. Under the action of the VBM shift, the electrons in Sb can be easily transferred to the conduction band of the $\mathrm{MoS}_{2}$ layer. At the same time, the holes of the $\mathrm{MoS}_{2}$ 
layer can be easily transferred to the valence band of the Sb layer. Due to the electron and hole transfer, the electron-hole pairs of the $\mathrm{Sb} / \mathrm{MoS}_{2}$ heterostructure decrease. This will effectively extend the life of the optoelectronics, thereby improving the performance of electronic and optoelectronic devices.

The band structures of $\mathrm{Sb} / \mathrm{MoS}_{2}$ superlattice are shown in Figure 2g,h. The superlattices with two different stackings are all shown to be direct band gap semiconductors. Compared with the hetero-bilayer $\mathrm{Sb} / \mathrm{MoS}_{2}$, the $\mathrm{CBM}$ of the $\mathrm{Sb} / \mathrm{MoS}_{2}$ superlattice is still all contributed by $\mathrm{MoS}_{2}$, while the VBM is occupied by Sb and $\mathrm{MoS}_{2}$. The total and partial DOS of $\mathrm{Sb} / \mathrm{MoS}_{2}$ superlattice are shown in Figure 2k,1. Within each monolayer of $\mathrm{MoS}_{2}$, there is strong hybridization between the Mo- $d$ orbitals and the S-s orbitals, and the Mo-S bond could preserve its covalent bonding. Compared with the Sb/MoS 2 hetero-bilayer (Figure 2i,j), we can clearly see that the contribution of antimonene in the valence band decreases, and the conduction band moves close to the Fermi level, leading to a decreased band gap in the $\mathrm{Sb} / \mathrm{MoS}_{2}$ superlattice. By controlling the number of layers in heterostructures, antimonene-based hybrid structures could realize a transformation from a type-I semiconductor to a type-II semiconductor. The results of this work provide a promising way to design applications in future antimonene-based nanodevices with controlled band gaps.

In order to better study the bonding nature and charge transfer of hybrid systems, we calculated the difference in charge density of planes perpendicular to the atomic layers and passing through Mo-S and Sb-Sb bonds (shown in Figure 3). The differential charge density $(\Delta \rho(\vec{r}))$ here is defined as:

$$
\Delta \rho(\vec{r})=\rho(\vec{r})-\sum_{\mu} \rho_{a t o m}\left(\vec{r}-\vec{R}_{\mu}\right)
$$

where $\rho(\vec{r})$ represents the total charge density of the heterostructure system and $\sum_{\mu} \rho_{a t o m}\left(\vec{r}-\vec{R}_{\mu}\right)$ is the superposition of atomic charge densities. The red solid line $(\Delta \rho(\vec{r})>0)$ and the blue dashed line $(\Delta \rho(\vec{r})<0)$ indicate the increase and decrease in the electron density after bonding, respectively. All the charge densities here are calculated using the same supercell for the heterostructures. As shown in Figure 3, within each atomic layer, the atomic bonding of Mo-S and Sb-Sb could preserve their covalent bonding, indicating that the interactions within each $2 \mathrm{D}$ sheet in heterostructures are not affected significantly by the introduction of foreign 2D materials. However, the weak interaction between layers will lead to a simple coupling effect between $\mathrm{Sb}$ and $\mathrm{MoS}_{2}$ layers. To clarify the specific reasons, the Bader analysis for the heterostructures was performed to calculate the charge transfer between the antimonene and $\mathrm{MoS}_{2}$ monolayer. According to the data in Table 2, we found that electrons are transferred from the $\mathrm{Sb}$ layer to the neighboring $\mathrm{MoS}_{2}$ layer (at the junction of $\mathrm{MoS}_{2}$ and $\mathrm{Sb}$ layers). However, the amount of charge transfer is very small (less than one electron). This charge is newly distributed after the formation of the $\mathrm{Sb} / \mathrm{MoS}_{2}$ heterostructures, which hinders the recombination of electron-hole pairs and balances the system.

Table 2. Bader analysis for the heterostructures.

\begin{tabular}{cccc}
\hline \multirow{2}{*}{ System } & & \multicolumn{2}{c}{ Gain/Loss Electrons } \\
\cline { 2 - 4 } & & Sb Layer & MoS $_{2}$ Layer \\
\hline \multirow{2}{*}{$\mathrm{Sb} / \mathrm{MoS}_{2}$ hetero-bilayer } & AC & -0.045 & 0.026 \\
\cline { 2 - 4 } & BC & -0.045 & 0.025 \\
\hline \multirow{2}{*}{$\mathrm{Sb} / \mathrm{MoS}_{2}$ superlattice } & AC & -0.099 & 0.056 \\
\cline { 2 - 4 } & $\mathrm{BC}$ & -0.098 & 0.055 \\
\hline
\end{tabular}



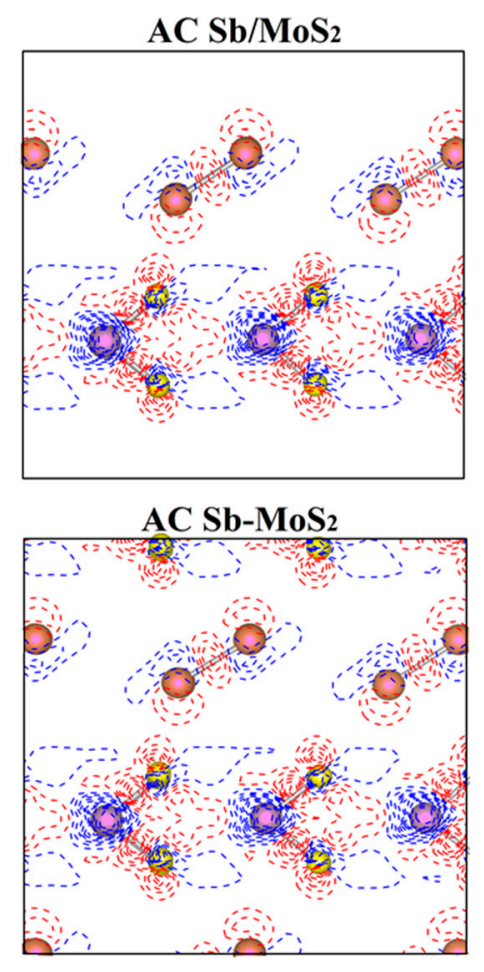

Figure 3. Deformation charge density $(\Delta \rho(\vec{r})$ for hybrid systems of planes perpendicular to the atomic layers and passing through Mo-S and Sb-Sb bonds. The red solid line and the blue dashed line indicate $\Delta \rho(\vec{r})<0$ and $\Delta \rho(\vec{r})<0$, respectively.

Compared to the isolated antimonene and $\mathrm{MoS}_{2}$ monolayer, the $\mathrm{Sb}$ and $\mathrm{MoS}_{2}$ layers in the hetero-bilayer have to be compressed or stretched to match each other. Thus, in order to get further insight into the effect of the in-plane biaxial strain on the hetero-bilayer systems, we systematically studied the electronic band structures and differential charge density of compressed (from $-1 \%$ to $-8 \%$ ) and stretched (from $1 \%$ to $4 \%$ ) $\mathrm{Sb} / \mathrm{MoS}_{2}$ hetero-bilayers. The in-plane biaxial strain $(\varepsilon)$ is defined as:

$$
\varepsilon=\frac{a-a_{0}}{a} \times 100 \%
$$

where $a$ and $a_{0}$ are the strained and unstrained lattice constants, respectively. The electronic band structures of the $\mathrm{Sb} / \mathrm{MoS}_{2}$ hetero-bilayer under different strains are shown in Figure 4, in which the band contribution from the antimonene and $\mathrm{MoS}_{2}$ monolayer are shown with blue and red dots, respectively. The band structure of the unstrained $\mathrm{Sb} / \mathrm{MoS}_{2}$ hetero-bilayer is also depicted in the middle of Figure 4 for comparison. For Sb/MoS 2 hetero-bilayers, the compressed (less than $-8 \%$ ) or stretched (less than $4 \%$ ) systems are still semiconductors with indirect band gaps. However, it can be seen that the value of band gaps can be varied by compressing or stretching the lattice constants (shown in Table 3). A maximum band gap of $1.00 \mathrm{eV}$ appears at the $\mathrm{Sb} / \mathrm{MoS}_{2}$ hetero-bilayer with a compressed strain of $-2 \%$. For stretched $\mathrm{Sb} / \mathrm{MoS}_{2}$ hetero-bilayers, the magnitude of band gaps decreases monotonically with an increase in lattice parameters. The size of the strain-induced band gap of $\mathrm{Sb} / \mathrm{MoS}_{2}$ hetero-bilayers is in the range of $0.10 \sim 1.00 \mathrm{eV}$. As the lattice constant is decreased, the band moves toward the deeper energy level. When a compressive strain of $-8 \%$ is applied, the $\mathrm{Sb} / \mathrm{MoS}_{2}$ hetero-bilayer is metallic. Hence, through compressing or stretching, the hetero-bilayers realize the transformation from a metal to an indirect band gap semiconductor. 


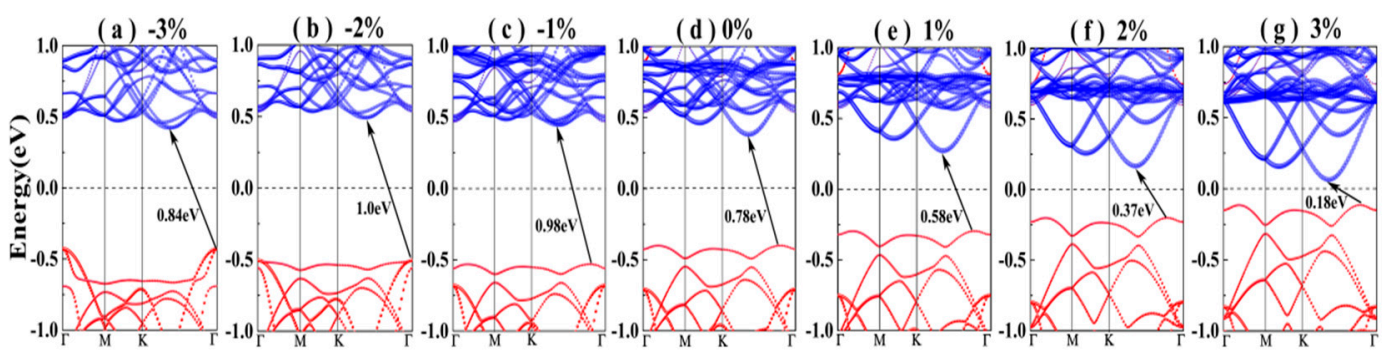

Figure 4. The electronic band structures of the $\mathrm{Sb} / \mathrm{MoS}_{2}$ hetero-bilayer under different in-plane biaxial strain: (a) $\varepsilon=-3 \%$, (b) $\varepsilon=-2 \%$, (c) $\varepsilon=-1 \%$, (d) $\varepsilon=0 \%$, (e) $\varepsilon=1 \%$, (f) $\varepsilon=2 \%$, (g) $\varepsilon=3 \%$. Contribution from antimonene and $\mathrm{MoS}_{2}$ monolayer to the band structures of the bilayers are shown with red and blue circle dots, respectively. The Fermi level is set to $0 \mathrm{eV}$.

Table 3. Band gaps of compressed/stretched $\mathrm{Sb} / \mathrm{MoS}_{2}$ hetero-bilayer.

\begin{tabular}{cccccccccccccc}
\hline strains & $-8 \%$ & $-7 \%$ & $-6 \%$ & $-5 \%$ & $-4 \%$ & $-3 \%$ & $-2 \%$ & $-1 \%$ & $0 \%$ & $1 \%$ & $2 \%$ & $3 \%$ & $4 \%$ \\
\hline band gap $(\mathrm{eV})$ & metal & 0.10 & 0.31 & 0.51 & 0.69 & 0.84 & 1.00 & 0.98 & 0.78 & 0.58 & 0.37 & 0.18 & metal \\
\hline
\end{tabular}

\section{Conclusions}

In summary, the electronic structures and properties of hybrid systems composed of antimonene and monolayer $\mathrm{MoS}_{2}$ were studied through density functional theory calculations. The $\mathrm{Sb} / \mathrm{MoS} 2$ hetero-bilayer and $\mathrm{Sb} / \mathrm{MoS}_{2}$ superlattice are both type-II band alignment, which implies the spatial separation of photogenerated electron-hole pairs. The $\mathrm{Sb} / \mathrm{MoS}_{2}$ hetero-bilayers are indirect band gap semiconductors, while the $\mathrm{Sb} / \mathrm{MoS}_{2}$ superlattices were shown to be direct band gap semiconductors with decreased band gaps. Due to the weak van der Waals interlayer interactions between layers, the electronic properties of each component can be preserved in the hybrid systems. In addition, the band gap of the $\mathrm{Sb} / \mathrm{MoS}_{2}$ hetero-bilayer can be effectively controlled by the biaxial strain, which improves its electronic performance in nanodevices. Our results show that antimonene-based hybrid systems are promising candidates for photovoltaic devices.

Author Contributions: Formal analysis, X.L.; funding acquisition, T.H.; investigation, C.Z.; project administration, X.L.; supervision, X.L.; writing—original draft, C.Z.; writing—review and editing, X.L. All authors have read and agreed to the published version of the manuscript.

Funding: This work is supported by the Fundamental Research Funds for the Central Universities, China under grant No. 2412019FZ037.

Conflicts of Interest: The authors declare no conflict of interest.

\section{References}

1. Xie, C.; Mak, C.H.; Tao, X.M.; Yan, F. Photodetectors Based on Dwo-Dimensional Layered Materials Beyond Graphene. Adv. Funct. Mater. 2017, 27, 1603886. [CrossRef]

2. Wu, J.B.; Lin, M.L.; Cong, X.; Liu, H.N.; Tan, P.H. Raman Spectroscopy of Graphene-Based Materials and Its Applications in Related Devices. Chem. Soc. Rev. 2018, 47, 1822-1873. [CrossRef] [PubMed]

3. Yu, X.W.; Cheng, H.H.; Zhang, M.; Zhao, Y.; Qu, L.T.; Shi, G.Q. Graphene-Based Smart Materials. Nat. Rev. Mater. 2017, 2, 17046. [CrossRef]

4. Lu, X.B.; Stepanov, P.; Yang, W.; Xie, M.; Aamir, M.A.; Das, I.; Urgell, C.; Watanabe, K.; Taniguchi, T.; Zhang, G.; et al. Superconductors, Orbital Magnets and Correlated States in Magic-Angle Bilayer Graphene. Nature 2019, 574, 653-657. [CrossRef]

5. Abraham, J.; Vasu, K.S.; Williams, C.D.; Gopinadhan, K.; Su, Y.; Cherian, C.; Dix, J.; Prestat, E.; Haigh, S.J.; Grigorieva, I.V.; et al. Tuneable Sieving of Ions Using Graphene Oxide Membranes. Nat. Nanotechnol. 2017, 12, 546-550. [CrossRef] 
6. Mo, R.W.; Rooney, D.; Sun, K.; Yang, H.Y. 3D Nitrogen-Doped Graphene Foam with Encapsulated Germanium/Nitrogen-Doped Graphene Yolk-Shell Nanoarchitecture for High-Performance Flexible Li-Ion Battery. Nat. Commun. 2017, 8, 13949. [CrossRef]

7. Li, X.L.; Zhi, L.J. Graphene Hybridization for Energy Storage Applications. Chem. Soc. Rev. 2018, 47, 3189-3216. [CrossRef]

8. Lee, I.H.; Yoo, D.; Avouris, P.; Low, T.; Oh, S.H. Graphene Acoustic Plasmon Resonator for Ultrasensitive Infrared Spectroscopy. Nat. Nanotech. 2019, 14,313-319. [CrossRef]

9. Geng, P.B.; Zheng, S.S.; Tang, H.; Zhu, R.M.; Zheng, L.; Cao, S.; Xue, H.G.; Pang, H. Transition Metal Sulfides Based on Graphene for Electrochemical Energy Storage. Adv. Energy Mater. 2018, 8, 1703259. [CrossRef]

10. Xu, Y.; Zhang, C.L.; Zhou, M.; Fu, Q.; Zhao, C.X.; Wu, M.H.; Lei, Y. Highly Nitrogen Doped Carbon Nanofibers with Superior Rate Capability and Cyclability for Potassium Ion Batteries. Nat. Commun. 2018, 9, 1720. [CrossRef]

11. Ma, J.; Liu, C.T. Using Negative Thermal Expansion Effect to Grow Spiny NiS/C Architecture and Its Application in Sodium-Ion Batteries. J. Alloys Compd. 2020, 829, 154572. [CrossRef]

12. Cai, Z.X.; Su, L.; Wang, H.J.; Niu, M.; Gao, H.F.; Lu, D.; Li, M.Z. Hydrophobic SiC@C Nanowire Foam with Broad-Band and Mechanically Controlled Electromagnetic Wave Absorption. ACS Appl. Mater. Interfaces 2020, 12, 8555-8562. [CrossRef] [PubMed]

13. Wong, K.L.; Chuan, M.W.; Hamzah, A.; Rusli, S.; Alias, N.; Sultan, M.S.; Lim, C.S.; Tan, M.L.T. Electronic Properties of Graphene Nanoribbons with Line-Edge Roughness Doped with Nitrogen and Boron. Physica E 2020, 117, 13841. [CrossRef]

14. Tang, S.B.; Wu, W.H.; Xie, X.J.; Li, X.K.; Gu, J.J. Band Gap Opening of Bilayer Graphene by Graphene Oxide Support Doping. RSC Adv. 2017, 7, 9862-9871. [CrossRef]

15. Yang, J.; Tang, L.S.; Bao, R.Y.; Bai, L.; Liu, Z.Y.; Xie, B.H.; Yang, M.B.; Yang, W. Hybrid Network Structure of Boron Bitride and Graphene Oxide in Shape-Stabilized Composite Phase Change Materials with Enhanced Thermal Conductivity and Light-to-Electric Energy Conversion Capability. Sol. Energy Mater. Sol. Cells. 2018, 174, 56-64. [CrossRef]

16. Ji, M.X.; Xia, J.X.; Di, J.; Liu, Y.L.; Chen, R.; Chen, Z.G.; Yin, S.; Li, H.M. Graphene-Like Boron Nitride Induced Accelerated Charge Transfer for Boosting the Photocatalytic Behavior of $\mathrm{Bi}_{4} \mathrm{O}_{5} \mathrm{I}_{2}$ Towards Bisphenol a Removal. Chem. Eng. J. 2018, 331, 355-363. [CrossRef]

17. Li, Q.; Huo, C.R.; Yi, K.; Zhou, L.L.; Su, L.; Hou, X.M. Preparation of Flake Hexagonal BN and Its Application in Electrochemical Detection of Ascorbic Acid, Dopamine and Uric Acid. Sens. Actuators B 2018, 260, 346-356. [CrossRef]

18. Zhao, J.; Li, N.; Yu, H.; Wei, Z.; Liao, M.Z.; Chen, P.; Wang, S.P.; Shi, D.X.; Sun, Q.J.; Zhang, G.Y. Highly Sensitive $\mathrm{MoS}_{2}$ Humidity Sensors Array for Noncontact Sensation. Adv. Mater. 2017, 29, 1702076. [CrossRef]

19. Liu, W.J.; Zhu, Y.N.; Liu, M.L.; Wen, B.; Fang, S.B.; Teng, H.; Lei, M.; Liu, L.M.; Wei, Z.Y. Optical Properties and Applications for $\mathrm{MoS}_{2}-\mathrm{Sb}_{2} \mathrm{Te}_{3}-\mathrm{MoS}_{2}$ Heterostructure Materials. Photonics Res. 2018, 6, 220-227. [CrossRef]

20. Sangeetha, M.; Madhan, D. Ultra Sensitive Molybdenum Disulfide $\left(\mathrm{MoS}_{2}\right) /$ graphene Based Hybrid Sensor for The Detection of $\mathrm{NO}_{2}$ and Formaldehyde Gases by Fiber Optic Clad Modified Method. Opt. Laser Technol. 2020, 127, 106193. [CrossRef]

21. Ares, P.; Aguilar-Galindo, D.; Rodriguez-San-Miguel, D.; Aldave, D.A.; Diaz-Tendero, S.; Alcami, M.; Martin, F.; Gomez-Herrero, J.; Zamora, F. Mechanical Isolation of Highly Stable Antimonene Under Ambient Conditions. Adv. Mater. 2016, 28, 6332-6336. [CrossRef] [PubMed]

22. Pumera, M.; Sofer, Z. 2D Monoelemental Arsenene, Antimonene, and Bismuthene: Beyond Black Phosphorus. Adv. Mater. 2017, 29, 1605299. [CrossRef] [PubMed]

23. Xie, M.Q.; Zhang, S.L.; Cai, B.; Gu, Y.; Liu, X.H.; Kan, E.J.; Zeng, H.B. Van der Waals Bilayer Antimonene: A Promising Thermophotovoltaic Cell Material With 31\% Energy Conversion Efficiency. Nano Energy 2017, 38, 561-568. [CrossRef]

24. Wang, X.X.; Tang, C.M.; Zhou, X.F.; Zhu, W.H.; Fu, L. The Good Performance of Bilayer $\beta$-Antimoneneas an Anode Material for the Li-ion Battery Study. Appl. Surf. Sci. 2019, 495, 143549. [CrossRef]

25. Zhang, J.J.; Zhang, Y.; Dong, S. Protective Layer Enhanced Stability and Superconductivity of Tailored Antimonene Bilayer. Phys. Rev. Mater. 2018, 2, 126004. [CrossRef] 
26. Alarawi, A.; Ramalingam, V.; Fu, H.C.; Purushothaman, V.; Yang, R.; He, J.H. Enhanced Photoelectrochemical Hydrogen Production Efficiency of $\mathrm{MoS}_{2}$-Si Heterojunction. Opt. Express 2019, 27, A352-A363. [CrossRef] [PubMed]

27. Qiao, S.; Cong, R.D.; Liu, J.H.; Liang, B.L.; Fu, G.S.; Yu, W.; Ren, K.L.; Wang, S.F.; Pan, C.F. A Vertically Layered $\mathrm{MoS}_{2} / \mathrm{Si}$ Heterojunction for an Ultrahigh and Ultrafast Photoresponse Photodetector. J. Mater. Chem. C 2018, 6, 3233-3239. [CrossRef]

28. Son, S.B.; Kim, Y.; Cho, B.; Choi, C.J.; Hong, W.K. Temperature-Dependent Electronic Charge Transport Characteristics at $\mathrm{MoS}_{2} / \mathrm{p}$-type Ge heterojunctions. J. Alloys Compd. 2018, 757, 221-227. [CrossRef]

29. Li, X.D.; Yu, S.; Wu, S.Q.; Zhou, S.; Zhu, Z.Z. Structural and Electronic Properties of Germanene/MoS 2 Monolayer and Silicene/MoS ${ }_{2}$ Monolayer Superlattices. Nanoscale Res. Lett. 2014, 9, 110. [CrossRef]

30. Wan, W.; Li, X.D.; Li, X.T.; Xu, B.B.; Zhan, L.J.; Zhao, Z.J.; Zhang, P.C.; Wu, S.Q.; Zhu, Z.Z.; Huang, H.; et al. Interlayer Coupling of a Direct Van der Waals Epitaxial $\mathrm{MoS}_{2}$ /graphene Heterostructure. RSC Adv. 2016, 6, 323-330. [CrossRef]

31. Li, X.D.; Yu, S.; Wu, S.Q.; Wen, Y.H.; Zhou, S.; Zhu, Z.Z. Structural and Electronic Properties of Superlattice Composed of Graphene and Monolayer MoS 2 . J. Phys. Chem. C 2013, 117, 15347-15353. [CrossRef]

32. Han, J.K.; Du, G.L.; Gao, W.W.; Bai, H. An Anisotropically High Thermal Conductive Boron Nitride/Epoxy Composite Based on Nacre-Mimetic 3D Network. Adv. Funct. Mater. 2019, 29, 1900412. [CrossRef]

33. Chittari, B.L.; Chen, G.R.; Zhang, Y.B.; Wang, F.; Jung, J. Gate-Tunable Topological Flat Bands in Trilayer Graphene-Boron Nitride Moire Superlattices. Phys. Rev. Lett. 2019, 122, 016401. [CrossRef] [PubMed]

34. Jiang, Y.Y.; Lin, X.; Low, T.; Zhang, B.L.; Chen, H.S. Group-Velocity-Controlled and Gate-Tunable Directional Excitation of Polaritons in Graphene-boron Nitride Heterostructures. Laser Photonics Rev. 2018, 12, 1800049. [CrossRef]

35. Laturia, A.; Van de Put, M.L.; Vandenberghe, W.G. Dielectric Properties of Hexagonal Boron Nitride and Transition Metal Dichalcogenides: From Monolayer to Bulk. NPJ 2D Mater. Appl. 2018, 2, 6. [CrossRef]

36. Pham, K.D.; Hieu, N.N.; Bui, L.M.; Phuc, H.V.; Hoi, B.D.; Tu, L.T.N.; Bach, L.G.; Ilyasov, V.V.; Amin, B.; Idress, M.; et al. Vertical Strain and Electric Field Tunable Electronic Properties of Type-II Band Alignment $\mathrm{C}_{2} \mathrm{~N} / \mathrm{InSe}$ Van der Waals Heterostructure. Chem. Phys. Leet. 2019, 716, 155-161. [CrossRef]

37. You, B.Q.; Wang, X.C.; Zheng, Z.D.; Mi, W.B. Black Phosphorene/Monolayer Transition-Metal Dichalcogenides as two Dimensional Van der Waals Heterostructures: A First-Principles Study. Phys. Chem. Chem. Phys. 2016, 18, 7381-7388. [CrossRef]

38. Zhang, F.; Li, W.; Dai, X.Q. Effects of Interlayer Coupling on the Electronic Structures of Antimonene/Graphene Wan der Waals Heterostructures. Superlattices Microstruct. 2016, 100, 826-832. [CrossRef]

39. Shen, N.F.; Yang, X.D.; Wang, X.X.; Wang, G.H.; Wan, J.G. Two-Dimensional Van der Waals Heterostructure of Indium Selenide/Antimonene: Efficient Carrier Separation. Chem. Phys. Lett. 2019, 727, 50-54. [CrossRef]

40. Sham, L.J.; Schlüter, M. Density-Functional Theory of the Energy Gap. Phys. Rev. Lett. 1983, 51, $1888-1891$. [CrossRef]

41. Kresse, G.; Furthmüller, J. Efficient Iterative Schemes for Ab Initio Total-Energy Calculations Using a Plane-Wave Basis Set. Phys. Rev. B 1996, 54, 11169-11186. [CrossRef] [PubMed]

42. Kresse, G.; Furthmüller, J. Efficiency of Ab-Initio Total Energy Calculations for Metals and Semiconductors Using a Plane-Wave Basis Set. Comput. Mater. Sci. 1996, 6, 15-50. [CrossRef]

43. Perdew, P.J.; Burke, K.; Ernzerhof, M. Generalized Gradient Approximation Made Simple. Phys. Rev. Lett. 1996, 77, 3865-3868. [CrossRef] [PubMed]

44. Perdew, P.J.; Zunger, A. Self-Interaction Correction to Density-Functional Approximations for Many-Electron Systems. Phys. Rev. B 1981, 23, 5048-5079. [CrossRef]

45. Grimme, S. Semiempirical GGA-Dype Density Functional Constructed with A Long-Range Dispersion Correction. J. Comput. Chem. 2006, 27, 1787-1799. [CrossRef] [PubMed]

46. Chadi, D.J. Special Points for Brillouin-Zone Integrations. Phys. Rev. B 1977, 16, 1746-1747. [CrossRef]

47. Ramírez, R.; Böhm, M.C. Simple Geometric Generation of Special Points in Brillouin-Zone Integrations. Two-Dimensional Bravais Lattices. Int. J. Quantum Chem. 1986, 30, 391-411. [CrossRef]

48. Chen, B.; Meng, Y.H.; Sha, J.W.; Zhong, C.; Hu, W.B.; Zhao, N.Q. Preparation of $\mathrm{MoS}_{2} / \mathrm{TiO}_{2}$ Based Nanocomposites for Photocatalysis and Rechargeable Batteries: Progress, Challenges, and Perspective. Nanoscale 2017, 10, 34-68. [CrossRef] 
49. Wang, S.; Ren, C.D.; Tian, H.Y.; Yu, J.; Sun, M.L. MoS 2 /ZnO Van der Waals Heterostructure as A High-Efficiency Water Splitting Photocatalyst: A First-Principles Study. Phys. Chem. Chem. Phys. 2018, 20, 13394-13399. [CrossRef]

50. Wu, X.; Shao, Y.; Liu, H.; Feng, Z.L.; Wang, Y.L.; Sun, J.T.; Liu, C.; Wang, J.Q.; Liu, Z.L.; Zhu, S.Y.; et al. Epitaxial Growth and Air-Stability of Monolayer Antimonene on PdTe 2 . Adv. Mater. 2017, 29, 1605407. [CrossRef]

51. Shao, Y.; LIiu, Z.L.; Cheng, C.; Wu, X.; Liu, H.; Liu, C.; Wang, J.O.; Zhu, S.Y.; Wang, Y.Q.; Shi, D.X.; et al. Epitaxial Growth of Flat Antimonene Monolayer: A New Honeycomb Analogue of Graphene. Nano Lett. 2018, 18, 2133-2139. [CrossRef] [PubMed]

52. Ares, P.; Palacios, J.J.; Abellan, G.; Gomez-Herrero, J.; Zamora, F. Recent Progress on Antimonene: A New Bidimensional Material. Adv. Mater. 2017, 30, 1703771. [CrossRef] [PubMed]

53. Chen, X.P.; Meng, R.S.; Jiang, J.K.; Liang, Q.H.; Yang, Q.; Tan, C.J.; Sun, X.; Zhang, S.L.; Ren, T.L. Electronic Structure and Optical Properties of Graphene/Stanene Heterobilayer. Phys. Chem. Chem. Phys. 2016, 18, 16302-16309. [CrossRef] [PubMed]

54. Águila, E.J.C.; Palacios, P.; Conesa, C.J.; Arriaga, J.; Wahnón, P. Electronic Band Alignment at CuGaS 2 Chalcopyrite Interfaces. Comput. Mater. Sci. 2016, 121, 79-85. [CrossRef]

55. Si, F.J.; Hu, W.; Tang, F.L.; Cheng, Y.W.; Xue, H.T. Electronic and Optical Properties of the Wurtzite-ZnO/CH $3 \mathrm{NH}_{3} \mathrm{PbI}_{3}$ Interface: First-Principles Calculations. J. Mater. Sci. 2017, 52, 13841-13851. [CrossRef]

Publisher's Note: MDPI stays neutral with regard to jurisdictional claims in published maps and institutional affiliations.

(C) 2020 by the authors. Licensee MDPI, Basel, Switzerland. This article is an open access article distributed under the terms and conditions of the Creative Commons Attribution (CC BY) license (http://creativecommons.org/licenses/by/4.0/). 